\title{
Synthesis of Artificial Magnetic Conductors Using Structure-Based Evolutionary Design
}

\author{
Luisa Deias, Giuseppe Mazzarella, Giorgio Montisci, and Giovanni Andrea Casula
}

Dipartimento di Ingegneria Elettrica ed Elettronica, Università di Cagliari, Piazza D’Armi, 09123 Cagliari, Italy

Correspondence should be addressed to Giuseppe Mazzarella; mazzarella@diee.unica.it

Received 10 October 2012; Revised 11 February 2013; Accepted 27 March 2013

Academic Editor: Karu P. Esselle

Copyright (C) 2013 Luisa Deias et al. This is an open access article distributed under the Creative Commons Attribution License, which permits unrestricted use, distribution, and reproduction in any medium, provided the original work is properly cited.

\begin{abstract}
An evolutionary programming approach, the so-called structure based evolutionary design, is applied to the synthesis of planar periodic electronic band gap in order to obtain an artificial magnetic conductor surface. We show that this strategy, in conjunction with a flexible aperture-oriented approach, allows for obtaining new and effective structures. This almost unique ability is exploited to obtain an artificial magnetic conductor periodic surface with a bandwidth larger than the most popular surfaces known so far.
\end{abstract}

\section{Introduction}

Frequency selective surfaces (FSS) consist of two-dimensional periodic arrays of metal patches patterned on a dielectric substrate or apertures etched on a metal screen [13]. These periodic structures resonate at certain frequencies, thus ensuring filtering characteristics, exploited both in the microwave and optical region of the electromagnetic spectrum. Frequency selective surfaces have been thoroughly studied over the years $[4,5]$, and they have found new life in the past decade when electromagnetic band gap (EBG) $[3,6]$ structures were introduced. Some well-known EBG structures are the uniplanar compact photonic band gap (UCPBG) proposed by Itoh [3] and the Sievenpiper "mushroom" high-impedance surface [6].

This class of structures, encompassing FSS as one of its subclasses (planar EBG), displays some very interesting electromagnetic properties. In particular, the presence of a stop band has been theoretically and experimentally verified and exploited in different realizations, that is, TEM waveguides, slow-wave planar structures, and low-loss conductor-backed coplanar waveguides. By choosing the proper geometry of a planar periodic surface we can shape the electromagnetic behavior of these structures.

We are interested here in planar periodic structures designed to act as an artificial magnetic conductor (AMC) or high-impedance electromagnetic ground plane over a desired (quite) narrow frequency range, corresponding to the forbidden frequency band. Hence, the key feature of these structures is the reflection of an incident plane wave with no phase reversal, unlike normal metal surfaces [6].

High-impedance surfaces have been widely studied as promising antenna substrates with either single-layer $[1,7,8]$ or double-layer [9] structure. A possibility of realizing a magnetic wall near the resonant frequency of a very thin structure is very attractive, since this allows one to design or enhance the performance of low-profile printed antennas. The main drawback of this strategy is the reduced bandwidth of the complete antenna, since the frequency range over which these EBG surfaces behave as an AMC is usually narrowband and fixed by their geometrical configuration. The ultimate goal, to effectively exploit the AMC in printed antennas, is to obtain an $\mathrm{AMC}$ with relatively large bandwidth lest the AMC bandwidth be the bottleneck of the antenna. Since the bandwidth of the AMC structures proposed so far cannot be further improved, an increase in bandwidth can only be obtained looking for different, and more performant, structures. A key issue in the research field of metamaterials is then represented by the design and optimization of new EBG structures. Different techniques have been investigated, in particular genetic algorithm (GA) and particle swarm optimization (PSO) [10-14]. However, all of them require a 
quite rigid specification of the structure that we are seeking. As a matter of fact, those approaches work on a (possibly large) number of numerical parameters but are unable to reach completely new structures.

This is, however, possible if a different point of view is taken, namely, SED (structure based evolutionary design). This approach, initially named genetic programming (GP), was first proposed by Koza [15] and is based on the idea that most real-world problems cannot be handled with parametric representations $[16,17]$. To summarize SED, we can say that it works on unambiguous structure descriptions represented as the set of instructions needed to realize it. Therefore, the approach proposed by Koza assumes no "a priori" structure. Instead, it builds up the structure of the individuals as the procedure evolves. As a consequence, its solution space encompasses every structure that can be realized and therefore has the power of the continuum, whereas the GA and PSO solution spaces are discrete ones, so they are a very small subspace of the former. As a consequence, the way the individuals (i.e., the actual structures) are represented in the SED procedure must be as effective as possible in order to explore its solution space at the best. A further specific point is the choice of the fitness function which strongly depends on the problem at hand. Its main role is to drive the procedure toward the optimal solution. As such, it must include the design specifications, but its formulation must be based on the structure behavior (in a broad sense). As a matter of fact, one of the main risks of every optimization procedure is the presence of traps (i.e., local optima). Different structures (wire-printed antennas, periodic AMC, etc.) show completely different routes to trap. The choice of the fitness function must also reflect this behavior, since another role of the fitness function is to prevent SED ending in a trap.

Another critical point can be present in SED. Since SED allows exploring and evaluating general configurations, it can lead to a severely ill-conditioned synthesis problem. As a consequence, a suitable stabilization is required and it can be obtained by imposing problem-specific requirements. We therefore let the SED strategy evaluate every possible geometry in the solution space constrained only by the previous requirements.

\section{A Brief SED Description}

A detailed description of SED is available elsewhere (see, e.g., $[15,18,19])$. We therefore make here a very brief introduction of SED and focus, in the next section, on the peculiar details of our implementation of SED for AMC design.

The evolutionary strategy works in order to find the best individuals, evaluated as those with the highest values of a suitable "fitness" function, expressing their closeness to the constraints set for the design.

Actually, the evolutionary "strategy" is almost standard for a variety of problems and is described in the flowchart of Figure 1. On the other hand, implementation of SED strategy is strictly related to the problem at hand and it cannot be simply reduced to a mere extension of what is already done for another class of problems. In order to take full advantage of the SED strategy, we therefore need to tailor its capabilities

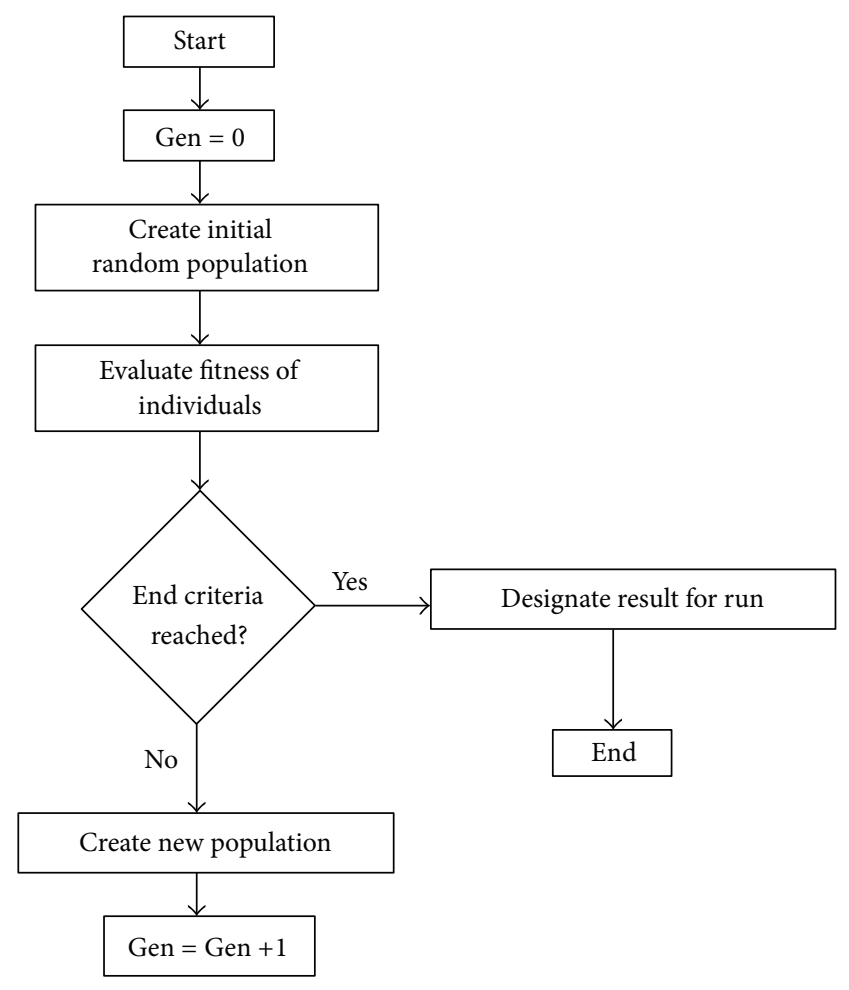

FIgURE 1: Flowchart of the evolutionary algorithm.

and behavior to the particular problem we are dealing with. Four elements must be defined as follows to effectively evolve a particular design:

(i) initial population;

(ii) representation scheme;

(iii) variation operators;

(iv) fitness function.

The starting point is an initial population of randomly generated computer programs composed of functions and terminals appropriate to the specified problem domain.

The initialization of an evolutionary algorithm can be completely at random or can incorporate human or other expertise about solutions that may work better than others.

Representation is a key issue in evolutionary programming approaches like SED [15] because the representation scheme can severely limit the window through which the system "observes" its world. From a general point of view, each individual is represented by a computer program, described through the set of instructions needed to "build" it, typically implemented in S-expressions (see the appendix).

Then, variation operations are applied to existing solutions to create new solutions. The role of these operators is to create new individuals from old ones; hence, their implementation is a code that takes one or more design representations as input and outputs a design derived from them.

Crossover. Create new S-expressions (the children) by exchanging sub-S-expressions between two S-expressions (the 
parents). The sub S-expressions exchanged are selected randomly.

Mutation. Creates a new S-expression from the parent one by replacing an existing argument symbol with another possible symbol. The argument symbol replaced is selected randomly.

The fitness criteria measure the quality of any givensolution, that is, its closeness to the set of design constraints and requirements. The selection method uses the score obtained for each solution to determine which to save and which to eliminate from the population at each generation. Those solutions that survive are the "parents" of the next generation, which is obtained by a random variation of the old one, using the variation operators. Such evolutionary pressure allows the geometrical properties of the structures under consideration to evolve towards the best solution, that is, a solution with a zero phase at the desired resonant frequency, and a rather large bandwidth.

At the end of the procedure, a full population of individual has been produced, all with a high fitness. The result of the design procedure is the best individual, for example, the one with the highest fitness. However, all other individuals of the final populations are available for considerations.

\section{Implementation of AMC Design Using SED}

The goal of the design process is to obtain the (aperture) geometry of the unit cell of the periodic surface which fulfills the desired requirements on the resonant frequency and bandwidth.

The periodic structure for each individual has been analyzed with an in-house full-wave analysis program, which is described and assessed in [20,21]. Our program is based on the method of moments $[22,23]$. It allows a time-effective solution and therefore is well suited for the needs of an evolutionary optimization approach. It has been fully validated through comparison with the Itoh structure and with a fullwave simulator, namely, Ansys HFSS, a finite element method general purpose software for the analysis of electromagnetic structures [24-26].

Therefore, we have chosen a representation able to describe a general structure while allowing an easy evaluation by our MoM code. A typical "individual" of our population is shown in Figure 2 and its internal S-expression is shown in the appendix. The chosen S-expression describes the geometry of the AMC cell as a set of rectangles, which is the input required by the MoM program (which uses a RWG discretization [27]).

Actually, the internal representation consists of a sequence of rectangles, each one starting at a vertex of the previous one, rotation operators, and "branching." The latter allows for two rectangles starting at the same point. Mutation operator allows for the change of rectangle size or angle of rotation. Crossover operator, on the other side, exchanges two complete subtrees, by exchanging their roots. In this way, borrowing the biological language, SED actually works at the organ level.

The center (resonant) frequency $f_{c}$ and the external parameters of the structure (i.e., substrate thickness and

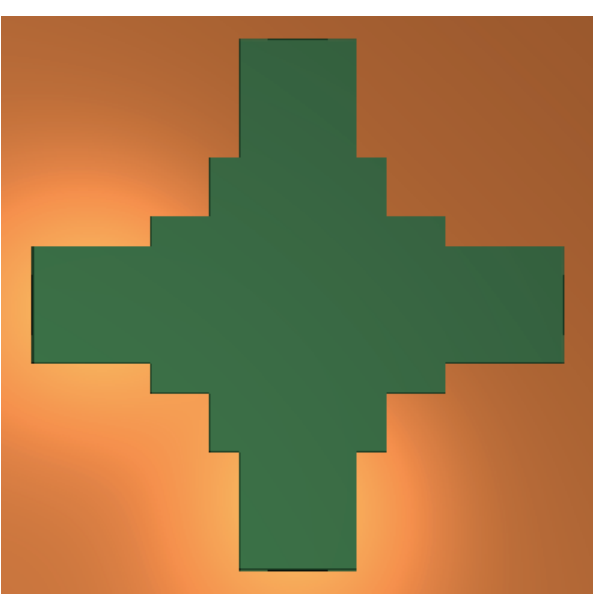

FIGURE 2: Aperture geometry.

dielectric permittivity) are fixed altogether with the periodicity of the planar AMC.

The set of admissible solutions is then composed of every geometry/aperture that can be designed in this square, built as series of segments that can evolve in every direction, with no limit on the number of segments, on their width and length and number of subsequent ramifications. However, we decided to evaluate only cells with quadrantal symmetry. Therefore, the internal representation of an individual describes only the first quadrant, and the MoM code "completes" the cell. We have fixed the discretization step within the unit cell in order to pose a limit not only to the computational burden but also to the geometrical spatial bandwidth of the possible solutions. In such a way, we obtain a stabilizing effect on the problem, as much as suggested (though for a different problem) in [28].

The phase of the reflection coefficient of the surface at the required resonant frequency and at the edges of the required bandwidth, as well as the geometrical data of the structure is the input to the fitness evaluation.

The fitness function employed by our SED optimization is

$$
\mathrm{FF}=\left(1+\omega_{1}\right) *\left(1+\omega_{2}\right) * e^{\operatorname{Phase}\left(f_{c}\right) / 20},
$$

where $\omega_{1}$ and $\omega_{2}$ are penalty coefficients developed in order to avoid geometries with high number of discretization elements within the unit cell and allowing for a large bandwidth centered on $f_{c}$. More precisely, $\omega_{1}$ is equal to zero when the number of the external edges of the RWG discretization [27] is smaller than given threshold; otherwise, $\omega_{1}$ is set to a quite large value. Instead, $\omega_{2}$ is proportional to the phase variation over the required bandwidth.

\section{Results}

Significative and promising results have been found when the approach described in Sections 2 and 3 is applied to a singlelayer EBG structure.

The structure data has been chosen as shown in Table 1. We have used a discretization step of $0.1016 \mathrm{~mm}$ for the unit 
TABLE 1: Fixed parameters of the periodic structure.

\begin{tabular}{lc}
\hline Center frequency & $14.2 \mathrm{GHz}$ \\
Periodicity (unit square cell) & $d_{x}=3.048 \mathrm{~mm}$ \\
& $d_{y}=3.048 \mathrm{~mm}$ \\
Substrate diel. constant & $\varepsilon_{r}=10.2$ \\
Substrate thickness & $0.635 \mathrm{~mm}$ \\
Discretization step & $0.1016 \mathrm{~mm}(30 \times 30$ grid $)$ \\
\hline
\end{tabular}

cell, making the playground for the SED optimization a $30 \times$ 30 grid in a square of $3.048 \mathrm{~mm}$ by $3.048 \mathrm{~mm}$. Such a choice allows for a direct comparison of the results of our procedure with one of the most popular AMC, namely the UC-EBG structure [3], shown in Figure 3 consisting of a Jerusalem cross aperture.

The full-wave analysis of the periodic structure has been performed by using our Fortran code $[20,21]$, which is independent of the particular shape of the metallization, which in the SED optimization stage cannot be known in advance.

The evolutionary strategy implementation chosen was ECJ, a general purpose Java-based Evolutionary Computation research system developed at ECLab (George Mason University) [29].

We have chosen population of 150 individuals, which means 150 runs of our executable, implementing the MoM evaluation, for each generation. The total computational burden associated with our executable is however dependent of the number of unknowns, which, in this case, are the internal edges of the discretized aperture. Therefore, the wider the aperture, the longer the analysis procedure will take.

Our MoM code evaluates each individual at the following frequencies: $f_{\min }, f_{c}$ (center frequency) and $f_{\max }$, wherein the shown cases $f_{\min }$ and $f_{\max }$ are chosen to be $1.5 \%$ of the center frequency. If the phase of the reflection coefficient at $f_{\min }$ and $f_{\max }$ has equal sign, then we are out of the resonant band; hence we chose $\omega_{1}$ to be a consistent penalty coefficient; that is, $\omega_{1}=10^{-2}$. When the phase at the two frequencies $f_{\min }$ and $f_{\max }$ has opposite sign, then we make $\omega_{1}$ a small coefficient penalty proportional to the phase difference $\Delta \Phi$ (in degrees), that is, $\omega_{1}=10^{-4} \Delta \Phi$. The coefficient can be further increased to obtain larger bandwidth structures. The coefficient penalty $\omega_{2}$ is associated with the number $N_{e}$ of internal edges, that is, of unknowns in our MoM evaluation problem. We decided to give a small and linear penalty to structures having a number of internal edges greater than 1000 in particular, we chose $\omega_{2}=10^{-12} N_{e}$.

First of all, we have required that the aperture geometry remains strictly inside the unit square cell. The penalty coefficients $\omega_{1}$ and $\omega_{2}$ in the fitness function (1) have been chosen to be quite loose, to allow a quick convergence toward the required AMC behavior at the center frequency. After a few generations, the last factor of (1) becomes close to 1 , and $\omega_{1}$ and $\omega_{2}$ start to drive the following subsequent generations toward simple and wideband structures.

The best individual, obtained after only 12 generations (and denoted by $\mathrm{A}$ in the following), is shown in Figure 4, together with a few previous generations. The phase at the

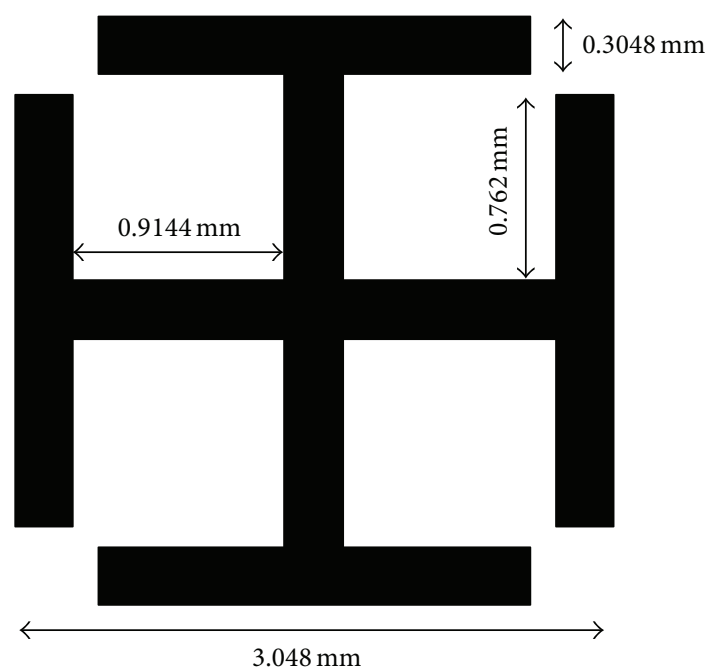

Figure 3: Reference UC-EBG geometry [3].

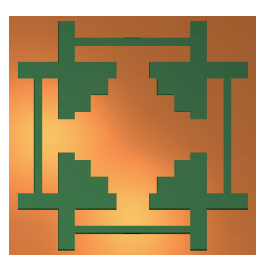

Generation 0

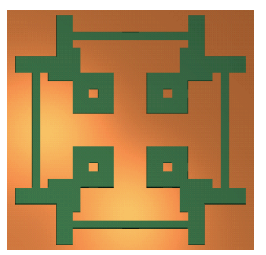

Generation 8

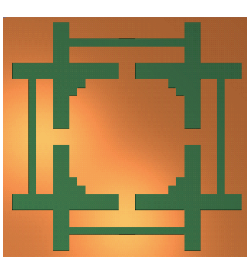

Generation 4

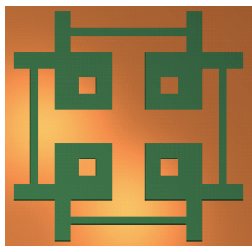

Generation 11

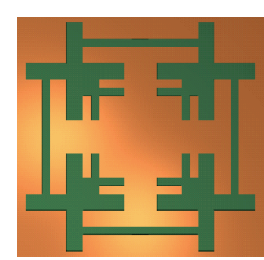

Generation 5

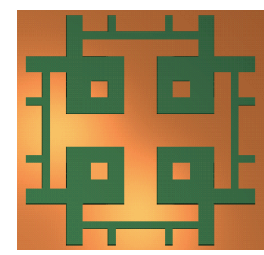

Generation 12
Figure 4: Evolution process-Case A.

center frequency $(14.2 \mathrm{GHz})$ is only $2.2^{\circ}$ with a bandwidth of $5 \%$ (comparable to the bandwidth of UC-PBG). This design has required 1800 evaluations of our in-house MoM code. Quite the same computational complexity has been required by all the subsequent examples.

The same requirements and data structure were kept for Case B, where we yet acted more stringently on the penalty coefficient $\omega_{1}$. In this case, the structure remains spatially more limited, as the number of unknowns is kept reasonably low, and the bandwidth is also improved. The number of generations in order to achieve a good result is still low (at gen. 11 the phase of the reflection coefficient at $14.2 \mathrm{GHz}$ is $\left.0.83^{\circ}\right)$.

In Case $\mathrm{C}$, while keeping the same data structure, we allowed the geometry to touch the borders of the unit cell, thus enlarging our solution space to consider also continuous aperture geometries. In this case, we have found a further and considerable improvement of the bandwidth. The convergence in this case is quite improved, and the structure 


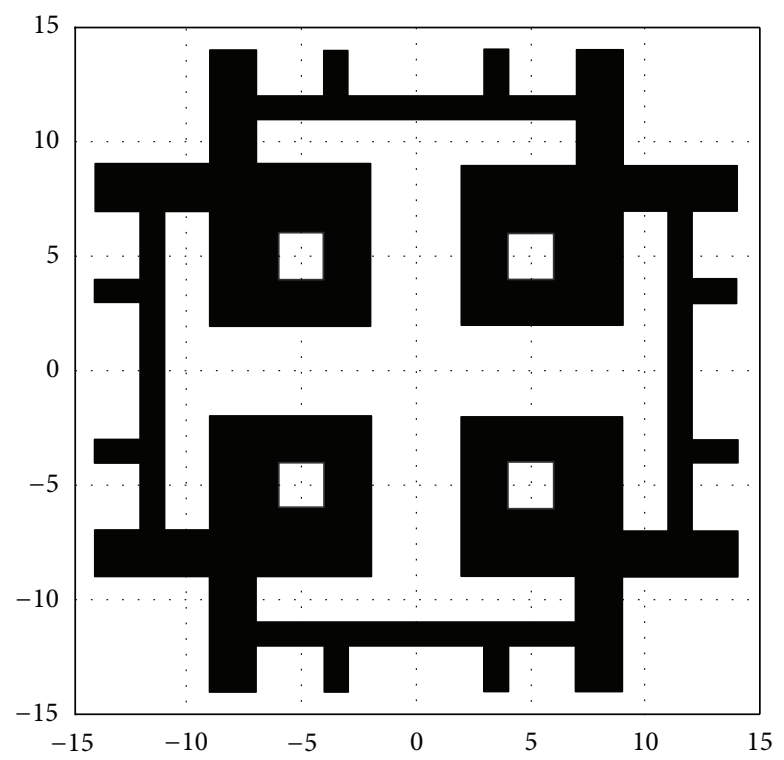

Figure 5: Best individual for Case A.

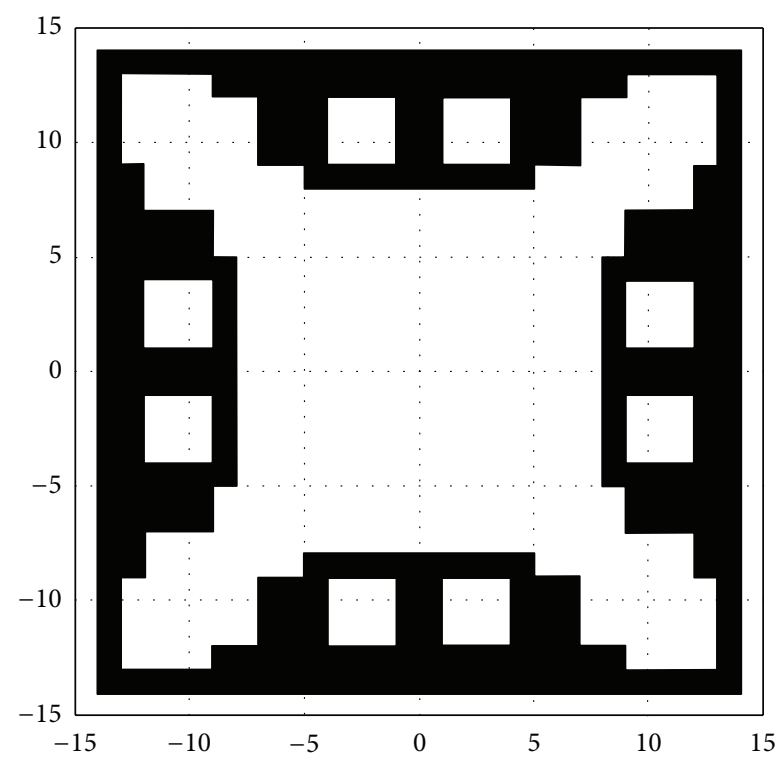

Figure 6: Best individual for Case B.

evolves even quickly towards the best individual (at gen. 8 the phase of the reflection coefficient at $14.2 \mathrm{GHz}$ is $10.5^{\circ}$ ).

In Figures 5, 6, and 7, we show the best individuals for each case. In Figure 8, the corresponding reflection coefficient is plotted, and a summary of the behavior of the AMC's designed with the proposed procedure is shown in Table 2. From a comparison with UC-PBG performance, also shown in Table 2, it is easy to see that the bandwidth of our designed $\mathrm{B}$ and $\mathrm{C}$ structures is around $8 \%$ and $15 \%$, respectively. The increase respect to UC-PBG, whose bandwidth (always computed as the frequency range in which the phase belongs to $\left.\left[-90^{\circ}, 90^{\circ}\right]\right)$ is around $5 \%$.

As expected, the geometries resemble the reference one and other well-known configurations, since the only stringent
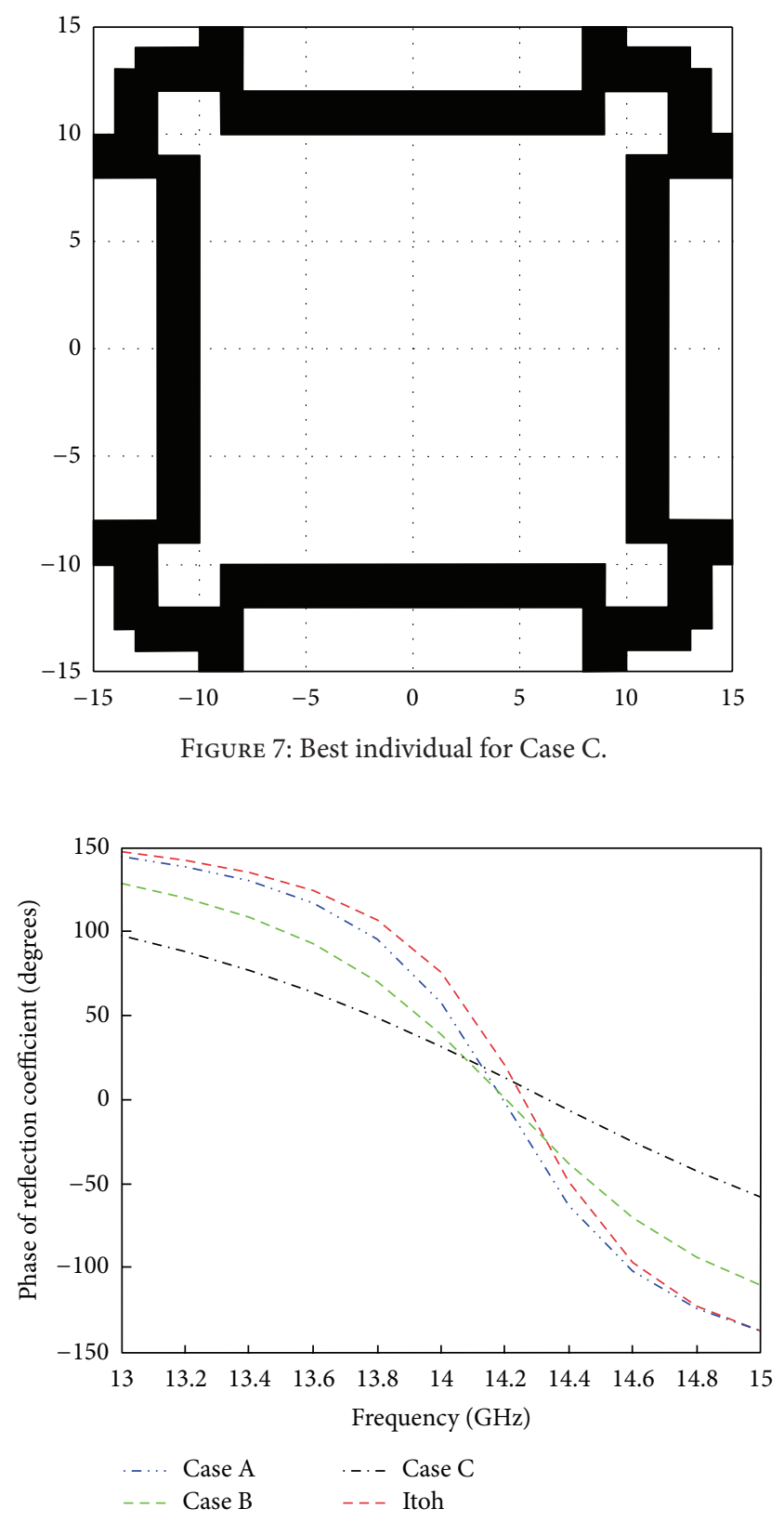

FIGURE 8: Reflection coefficient.

requirement in this case is the resonant frequency. The fitness in this case is relatively simple, aimed almost uniquely to direct the evolution process towards a structure that resonates at the desired frequency. Then, we can insert an additional penalty coefficient in order to maximize the bandwidth, a secondary objective overlapped to the main goal.

We have also tried different discretization steps within the unit cell and, as expected, we have found that small values can increase the probability that the fitness generates local minima/maxima, which traps the evolution process.

In order to evaluate our approach, we have tested it also using a few different requirements. Since it is well known that there is a growing interest in antennas integrated with EBG surfaces for communication system applications, covering 


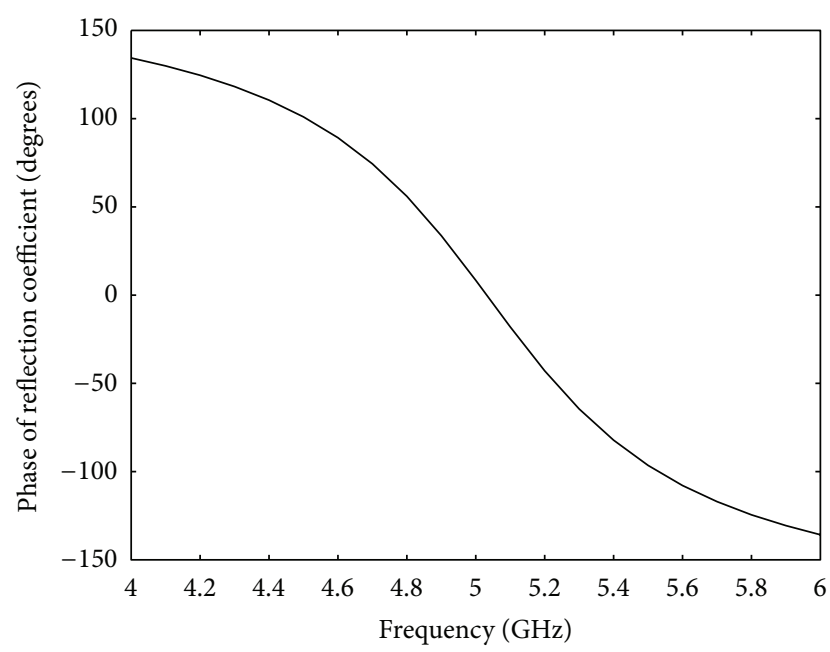

FIgUre 9: Reflection coefficient for the $5 \mathrm{GHz}$ EBG.

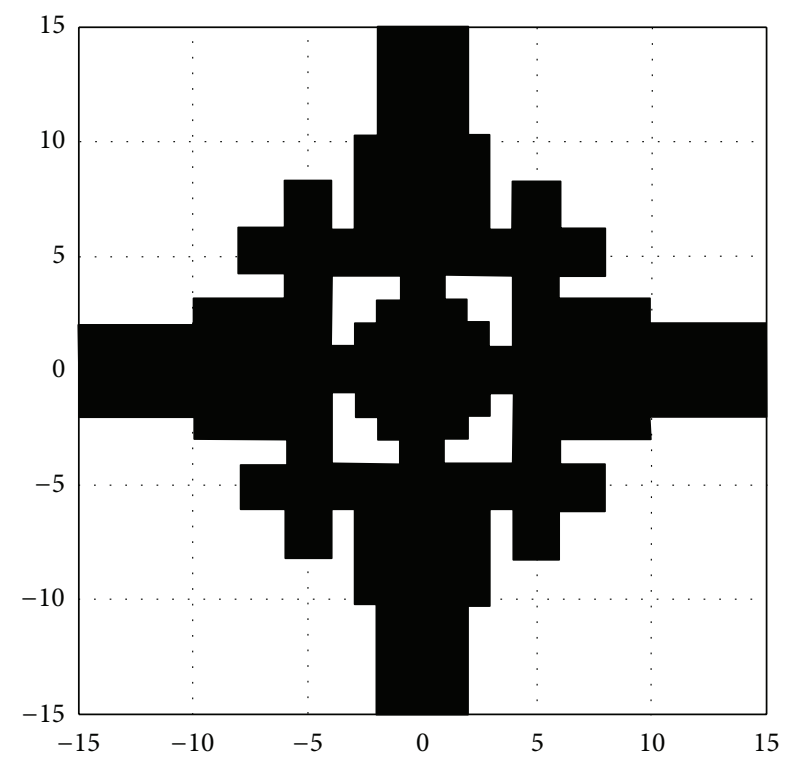

FIGURE 10: Geometry of the best individual for the $5 \mathrm{GHz}$ EBG.

TABLE 2: Comparison between the periodic structures derived from the best individuals shown in Figures 5, 6, and 7.

\begin{tabular}{lccc}
\hline & $N_{G}$ & $\Phi @ 14.2 \mathrm{GHz}$ & $B\left[-90^{\circ}: 90^{\circ}\right]$ \\
\hline Yang et al. [3] & - & $20.52^{\circ}$ & $13.9 \mathrm{GHz}-14.56 \mathrm{GHz}$ \\
Case A & 12 & $2.2^{\circ}$ & $13.8 \mathrm{GHz}-14.52 \mathrm{GHz}$ \\
Case B & 11 & $0.83^{\circ}$ & $13.6 \mathrm{GHz}-14.75 \mathrm{GHz}$ \\
Case C & 8 & $10.5^{\circ}$ & $13.1 \mathrm{GHz}-15.4 \mathrm{GHz}$ \\
\hline
\end{tabular}

$N_{G}$ : number of generation required to reach the best individual; $\Phi$ : phase of the reflection coefficient; $B$ : frequency bandwidth for $\Phi \in\left[-90^{\circ}: 90^{\circ}\right]$.

the wireless networking bands [30,31], we have considered the design of a single-layer AMC with a resonant frequency of $5 \mathrm{GHz}$. Fixed parameters for this case are shown in Table 3.

In Figure 9, we can see the reflection coefficient for the best individual shown Figure 10, as obtained after twelve generations. The performance of our optimization is quite good
TABLE 3: Fixed parameters of the periodic structure in the Sfrequency band.

\begin{tabular}{lc}
\hline Center frequency & $5 \mathrm{GHz}$ \\
Periodicity (unit square cell) & $d_{x}=25.908 \mathrm{~mm}$ \\
Substrate diel. constant & $d_{y}=25.908 \mathrm{~mm}$ \\
Substrate thickness & $\varepsilon_{r}=1.38$ \\
Discretization step & $2.2 \mathrm{~mm}$ \\
\hline
\end{tabular}

leading to a simple and effective solution with a bandwidth of about $20 \%$.

\section{Conclusions}

An evolutionary design strategy for Artificial Magnetic Conductors has been described and assessed for single-layers structures. The presented results show both the flexibility of the strategy, as well its ability to design wide-band AMC with a relatively reduced computational burden.

\section{Appendix}

The S-expression used in SED must be built to describe the way the individual is realized. In this work, each individual is composed of a number of metallic rectangles, which can be drawn toward either side of each of the two axes. Therefore, each S-expression is composed of the following objects (or instructions).

Rectangle which draws a rectangle of the specified size, toward the present direction and starting from a given point.

Rotate which simply rotates the present drawing direction (in our case, only by $\pm 90^{\circ}$ ).

Branch which allows that two objects start from the same point.

Rectangles of an S-expression can overlap, and, in this case, only one is considered and will be cut at the boundary.

In this work, we consider structures made by more than a single S-expression. For example, an actual S-expression considered in this work is as the following:

Tree 0:

(Branch

(Rectangle 0.165890 .28733 (Rotate 90.0 (Rotate -90.0 END)))

(Rotate 90.0 (Rectangle 0.683760 .27571 (Rectangle 0.725420 .23175

(Rotate 90.0 (Rotate 90.0 (Rotate -90.0 (Rectangle $0.1947(0.19593 \mathrm{END}))))))$ ) )

Tree 1:

(Branch (Branch END END) (Rotate 90.0 END))

Tree 2:

(Branch END END)

Tree 3:

(Rotate 90.0 (Rectangle 0.51570 .25133 (Rotate 90.0

(Branch

(Rotate -90.0 END)

(Rectangle 0.752310 .28339 END)))

) ) 
In this case, we have four "trees", that is, the metallization of this individual consists of the union of four polyrectangles.

This representation is actually redundant, but it is this redundancy that, as in the inspiring natural selection, is the key to different requirements and to escape more easily from traps. A sequence of two opposite rotations cannot be simply dropped, lest the variation is severely reduced. Actually, each of the two rotations can mutate or be a crossover point. However, if they are dropped, that part of the individual cannot be further modified by SED.

\section{References}

[1] M. Hosseini, A. Pirhadi, and M. Hakkak, "Design of a novel AMC with little sensitivity to the angle of incidence and very compact size," in Proceedings of the IEEE Antennas and Propagation Society International Symposium (APS '06), pp. 1939-1942, July 2006.

[2] T. K. Wu, Frequency Selective Surface and Grid Array, Wiley, New York, NY, USA, 1999.

[3] F. R. Yang, K. P. Ma, and Y. Qian, "A novel tem waveguide using uniplanar compact photonic-bandgap (uc-pbg) structure," IEEE Transactions on Microwave Theory and Techniques, vol. 47, no. 11, pp. 2092-2098, 1999.

[4] R. Mittra, C. H. Chan, and T. Cwik, “Techniques for analyzing frequency selective surfaces-a review," Proceedings of the IEEE, vol. 76, no. 12, pp. 1593-1615, 1988.

[5] B. Munk, Frequency Selective Surfaces: Theory and Design, Wiley Interscience, New York, NY, USA, 2000.

[6] D. Sievenpiper, L. Zhang, R. F. Broas, N. G. Alexöpolous, and E. Yablonovitch, "High-impedance electromagnetic surfaces with a forbidden frequency band," IEEE Transactions on Microwave Theory and Techniques, vol. 47, no. 11, pp. 2059-2074, 1999.

[7] A. P. Feresidis, G. Goussetis, S. Wang, and J. C. Vardaxoglou, "Artificial magnetic conductor surfaces and their application to low-profile high-gain planar antennas," IEEE Transactions on Antennas and Propagation, vol. 53, no. 1 I, pp. 209-215, 2005.

[8] R. Gonzalo, P. de Maagt, and M. Sorolla, "Enhanced patch-antenna performance by suppressing surface waves using photonic-bandgap substrates," IEEE Transactions on Microwave Theory and Techniques, vol. 47, no. 11, pp. 2131-2138, 1999.

[9] Y. Ranga, L. Matekovits, K. P. Esselle, and A. R. Weily, "Multioctave frequency selective surface reflector for ultrawideband antennas," IEEE Antennas and Wireless Propagation Letters, vol. 10, pp. 219-222, 2011.

[10] M. G. Bray, Z. Bayraktar, and D. H. Werner, "GA optimized ultra-thin tunable EBG AMC surfaces," in Proceedings of the IEEE Antennas and Propagation Society International Symposium (APS '06), pp. 410-413, July 2006.

[11] Y. Ge, K. P. Esselle, and Y. Hao, "Design of low-profile highgain EBG resonator antennas using a genetic algorithm," IEEE Antennas and Wireless Propagation Letters, vol. 6, pp. 480-483, 2007.

[12] P. Kovács, Z. Raida, and Z. Lukeš, "Design and optimization of periodic structures for simultaneous EBG and AMC operation," in Proceedings of the 15th Conference Microwave Techniques (COMITE '10), pp. 195-198, April 2010.

[13] A. A. Tavallaee and Y. Rahmat-Samii, "A novel strategy for broadband and miniaturized EBG designs: hybrid MTL theory and PSO algorithm," in Proceedings of the IEEE Antennas and
Propagation Society International Symposium (APS '07), pp. 161164, June 2007.

[14] J. Yeo, J. F. Ma, and R. Mittra, "Design of artificial magnetic ground planes (AMGs) utilizing frequency selective surfaces embedded in multilayer structures with electric and magnetic losses," in Proceedings of the IEEE Antennas and Propagation Society International Symposium (APS '03), vol. 3, pp. 343-346, June 2003.

[15] J. R. Koza, Genetic Programming: On the Programming of Computers by Means of Natural Selection, MIT Press, Cambridge, Mass, USA, 1992.

[16] D. Fogel, Evolutionary Computation: Toward a New Philosophy of Machine Intelligence, IEEE Press, New York, NY, USA, 2006.

[17] Z. Michalewicz, Genetic Algorithms + Data Structures = Evolution Programs, Springer, Berlin, Germany, 1992.

[18] G. A. Casula, G. Mazzarella, and N. Sirena, "Evolutionary design of wide-band parasitic dipole arrays," IEEE Transactions on Antennas and Propagation, vol. 59, no. 11, pp. 4094-4102, 2011.

[19] G. A. Casula, G. Mazzarella, and G. Montisci, "Structure-based evolutionary programming design of broadband wire antennas," International Journal of Antennas and Propagation, vol. 2013, Article ID 945408, 8 pages, 2013.

[20] L. Deias and G. Mazzarella, "Aperture oriented approach to the analysis of artificial magnetic conductors and first-order circuit model validation," in Proceedings of the 12th International Symposium on Antenna Technology and Applied Electromagnetics (ANTEM) and Canadian Radio Sciences (URSI/CNC), Montreal, Canada, July 2006.

[21] F. Asole, L. Deias, and G. Mazzarella, "A flexible full-wave analysis of multilayered AMC using an aperture oriented approach," Journal of Electromagnetic Waves and Applications, vol. 21, no. 14, pp. 2059-2072, 2007.

[22] G. Mazzarella and G. Montisci, "Accurate modeling of coupling junctions in dielectric covered waveguide slot arrays," Progress in Electromagnetics Research M, vol. 17, pp. 59-71, 2011.

[23] Z. Jin, G. Montisci, G. A. Casula, H. Yang, and J. Lu, "Efficient evaluation of the external mutual coupling in dielectric-covered waveguide slot arrays," International Journal of Antennas and Propagation, vol. 2012, Article ID 491242, 7 pages, 2012.

[24] G. Montisci, "Design of circularly polarized waveguide slot linear arrays," IEEE Transactions on Antennas and Propagation, vol. 54, no. 10, pp. 3025-3029, 2006.

[25] G. A. Casula and G. Montisci, "Design of dielectric-covered planar arrays of longitudinal slots," IEEE Antennas and Wireless Propagation Letters, vol. 8, pp. 752-755, 2009.

[26] S. Costanzo, G. A. Casula, A. Borgia et al., "Synthesis of slot arrays on integrated waveguides," IEEE Antennas and Wireless Propagation Letters, vol. 9, pp. 962-965, 2010.

[27] S. M. Rao, D. R. Wilton, and A. W. Glisson, "Electromagnetic scattering by surface of arbitrary shape," IEEE Transactions on Antennas and Propagation, vol. 30, no. 3, pp. 409-419, 1982.

[28] R. E. Collin, Antennas and Radiowave Propagation, section 2.12, McGraw-Hill, New York, NY, USA, 1985.

[29] http://cs.gmu.edu/ eclab/projects/ecj/ .

[30] H. H. Lin, C. Y. Wu, and S. H. Yeh, "Metamaterial enhanced high gain antenna for WiMAX application," in Proceedings of the IEEE Region 10 Conference (TENCON '07), Taipei, Taiwan, November 2007.

[31] S. Zhu and R. Langley, "Dual-band wearable textile antenna on an EBG substrate," IEEE Transactions on Antennas and Propagation, vol. 57, no. 4, pp. 926-935, 2009. 

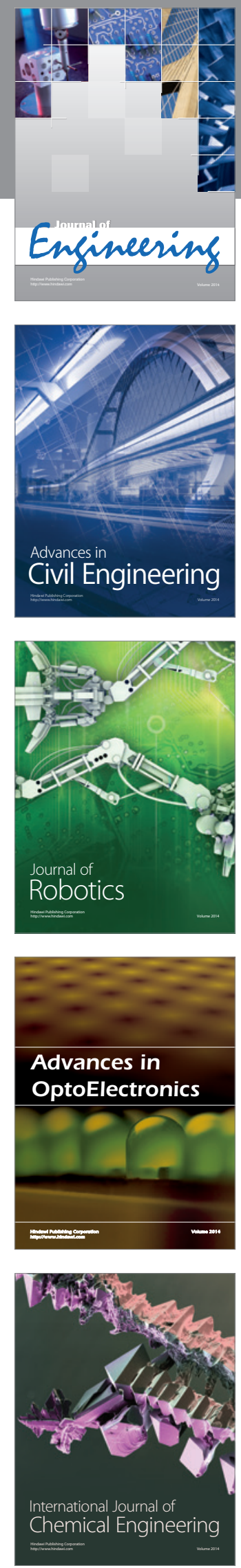

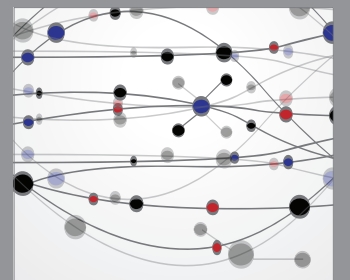

The Scientific World Journal
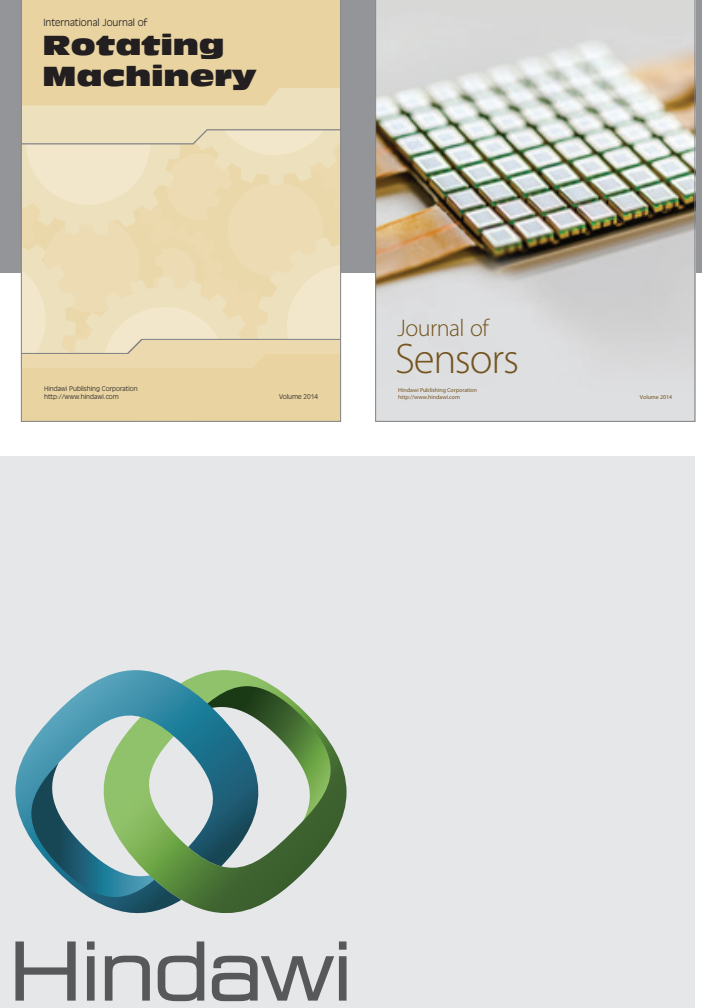

Submit your manuscripts at http://www.hindawi.com
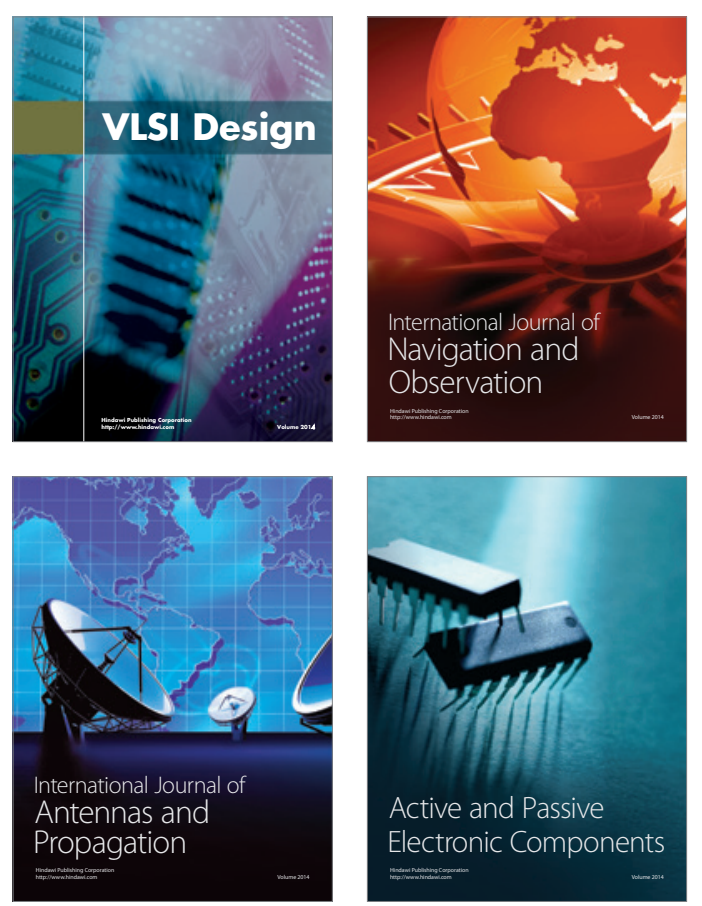
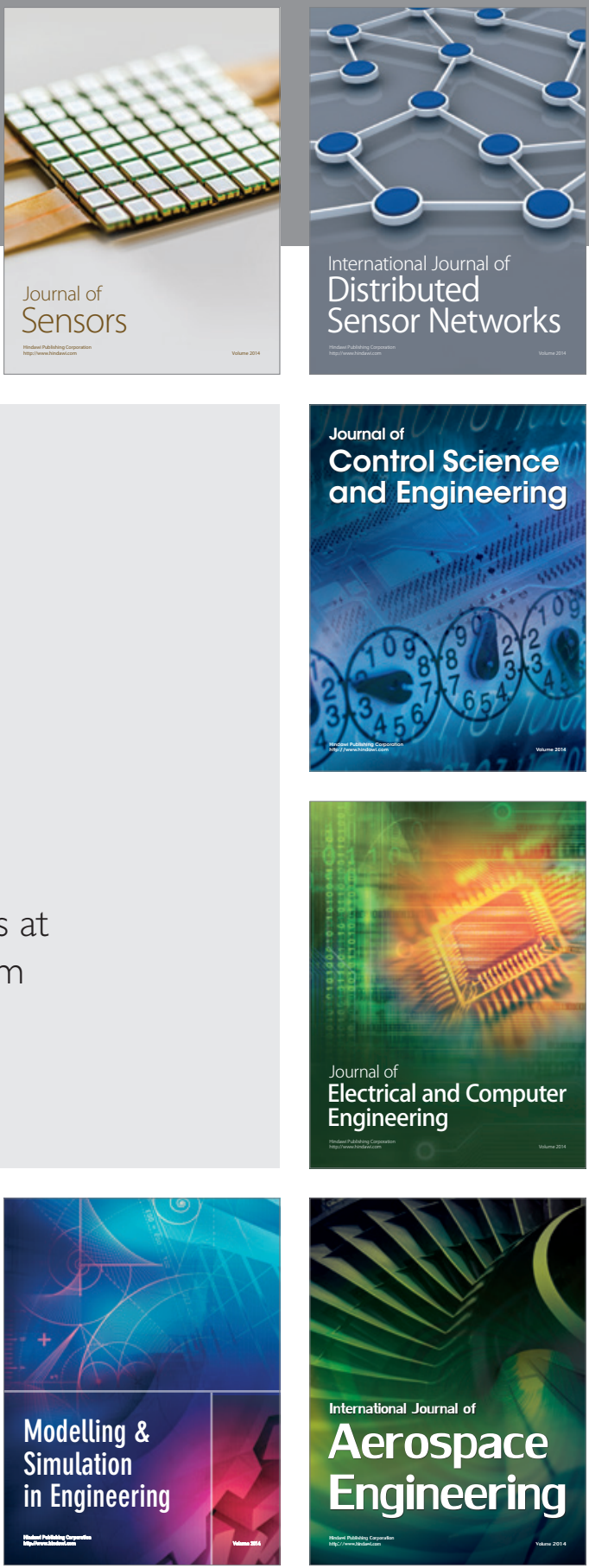

Journal of

Control Science

and Engineering
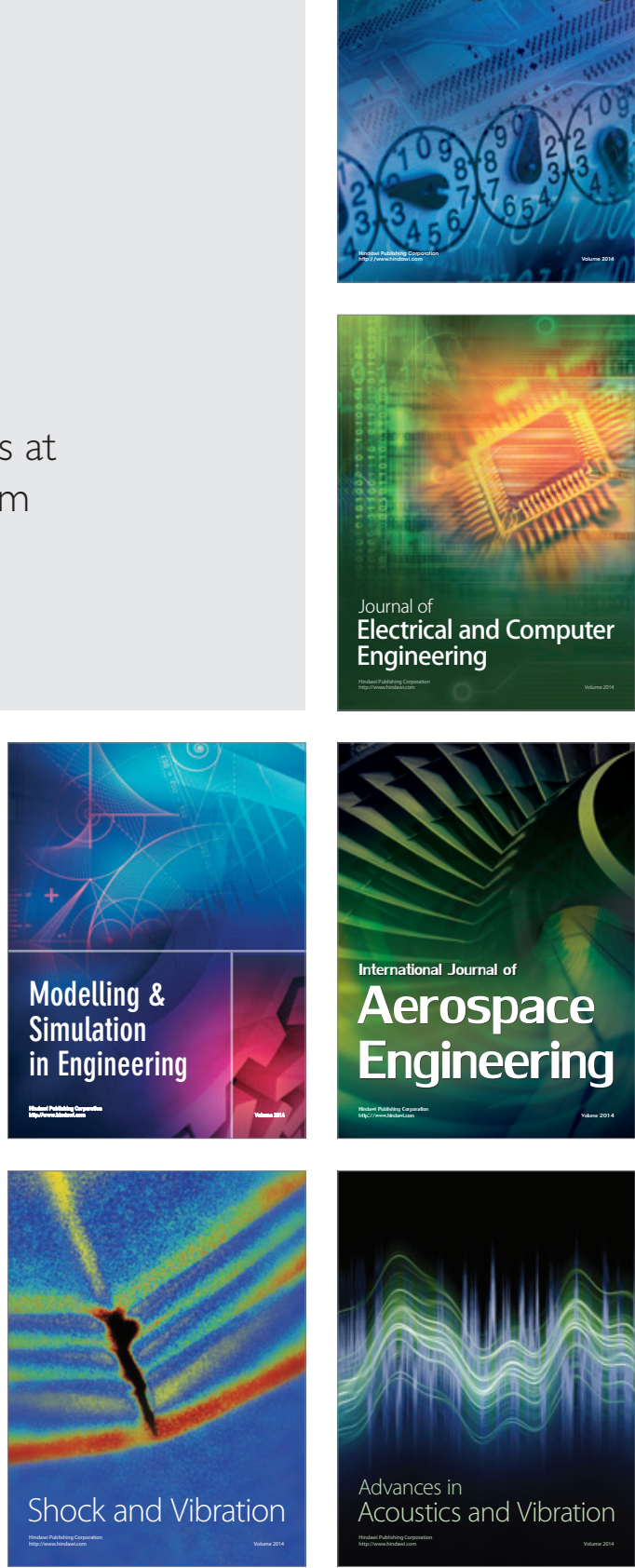